\title{
Publisher Correction: Amplification of intense light fields by nearly free electrons
}

Mary Matthews (iD, Felipe Morales (D), Alexander Patas, Albrecht Lindinger, Julien Gateau, Nicolas Berti, Sylvain Hermelin, Jérôme Kasparian (D), Maria Richter, Timm Bredtmann, Olga Smirnova (D), Jean-Pierre Wolf and Misha Ivanov

Correction to: Nature Physics https://doi.org/10.1038/s41567-018-0105-0, published online 16 April 2018.

In the version of this Letter originally published, the units of the bottom three values in the Fig. 1d legend were incorrect; they should have been $\mathrm{W} \mathrm{cm}^{-2}$. This has now been corrected.

\section{Author Correction: Quantum electrodynamics and the proton size}

Thomas Udem

Correction to: Nature Physics https://doi.org/10.1038/s41567-018-0166-0, published online 4 June 2018.

In the version of this Measure for Measure originally published, the discrepancy in the fifth paragraph was incorrectly written as $7 \sigma$; it should have been $5.6 \sigma$. This has now been corrected. 